\title{
Effect of Filter for Speed Servo Systems with PID Controller
}

\author{
Member Guoguang Zhang (Osaka University) \\ Non-member Junji Furusho (Osaka University)
}

Key words: Extended Parameter Plane Method, PID Control, Lowpass Filter, Two-inertia System.

\section{Introduction}

The servo control systems driving a load through a flexible shaft or transmission system are widely used in industrial applications. It was shown that PID control is able to provide an effective damping for speed control of two-mass system ${ }^{(1)(2)(3)(4)}$. In practice, the derivative feedback is usually carried out through a lowpass filter. Matsui presented the pole assignment with considering the filter ${ }^{(5)}$, and the time constant of the filter was treated as a free parameter.

In many industrial applications, the time constant of the filter is not a simple free-parameter since it is mainly determined by noises lever and high resonance modes. Purpose in this paper is to investigate the effect of the filter for speed servo systems with PID controller.

\section{Speed Control System}

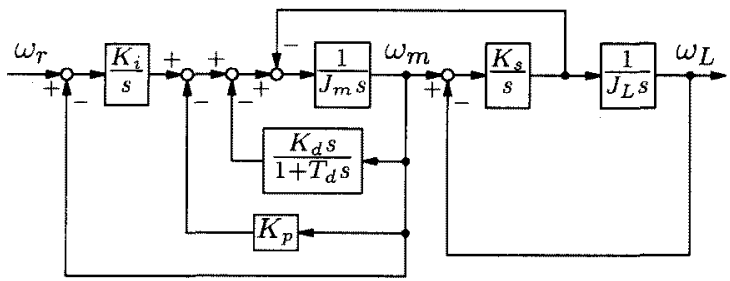

Fig. 1. A speed control system with PID controller plus a lowpass filter

A speed control system of two-inertia system, which employs PID control plus a lowpass filter, is shown in Fig.1. In this figure, $J_{m}$ : motor inertia, $J_{L}$ : load inertia, $\omega_{m}$ : motor speed, $\omega_{L}$ : load speed, $K_{s}$ : torsional stiffness of drive shaft, $\omega_{r}$ : reference input of motor speed. $K_{p}, K_{i}, K_{d}$ are proportional, integral and derivative feedback coefficients, respectively. $T_{d}$ is the time constant of the filter, then $1 / T_{d}$ corresponds to the bandwidth of the filter.
The transfer function from the reference input to the load speed is given by

$$
\frac{\omega_{L}(s)}{\omega_{r}(s)}=\frac{K_{i}\left(1+T_{d} s\right) \omega_{a}^{2}}{a_{5} s^{5}+a_{4} s^{4}+a_{3} s^{3}+a_{2} s^{2}+a_{1} s+a_{0}}
$$

where

$$
\begin{aligned}
& a_{5}=T_{d} \\
& a_{4}=1+T_{d} K_{p} / J_{m}+K_{d} / J_{m} \\
& a_{3}=\omega_{0}^{2} T_{d}+K_{p} / J_{m}+T_{d} K_{i} / J_{m} \\
& a_{2}=\omega_{0}^{2}+\omega_{a}^{2} T_{d} K_{p} / J_{m}+K_{i} / J_{m}+\omega_{a}^{2} K_{d} / J_{m} \\
& a_{1}=\omega_{a}^{2} K_{p} / J_{m}+\omega_{a}^{2} T_{d} K_{i} / J_{m} \\
& a_{0}=\omega_{a}^{2} K_{i} / J_{m}
\end{aligned}
$$

$\omega_{a}$ is antiresonant frequency, $\omega_{0}$ is resonant frequency, and $R$ is inertia ratio. They are defined as follows:

$$
\omega_{a}=\sqrt{\frac{K_{s}}{J_{L}}}, \quad \omega_{0}=\omega_{a} \sqrt{1+R}, \quad R=\frac{J_{L}}{J_{m}}
$$

The system in Eq.(1) is a fifth-order one, which has only three adjustable feedback coefficients $\left(K_{p}, K_{i}, K_{d}\right)$. It is not easy to design the system due to the less adjustable parameters. The extended parameter plane method proposed by authors is applied ${ }^{(B)}$.

The coefficients $a_{k}(k=0,1, \cdots, 5)$ in Eq.(1) are the linear functions of the three feedback parameters $K_{p}, K_{i}$ and $K_{d}$. Furthermore, $K_{p} / J_{m}, K_{i} / J_{m}$ and $K_{d} / J_{m}$ are considered as the three designed parameters in analysis since $J_{m}$ is a constant.

\section{System Analysis}

The extended parameter plane technique determines three adjustable parameter by assigning three poles in the complex plane ${ }^{(6)}$. It is understood from our previous work that the PID controller is very necessary for the small inertia ratio ${ }^{(1)}$. Here, the cases of the inertia ratio $R=0.5,0.75$ or 1.0 are analyzed. 
The effect on the bandwidth of the filter $\left(1 / T_{d}\right)$ is investigated. When $1 / T_{d}$ is set as $3 \omega_{a}$ or $5 \omega_{a}$, the pole locations are shown in Figs.2,3. In the two figures, the pair of the dominant poles $(\bullet)$ are fixed with the damping coefficient $=0.8$ and the natural frequency $=0.6 \omega_{a}$, but the real pole $(+)$ is changed from $-0.1 / T_{d}$ to $-0.6 / T_{d}$. It is shown that the locations of the real pole $(+)$ clearly effect the pair of vibratory poles $(x)$, and the system shows a slight vibratory behavior as the inertia ratio is lowered. It is also noted that the damping of the poles $(x)$ is improved as the bandwidth of the filter gets wider.

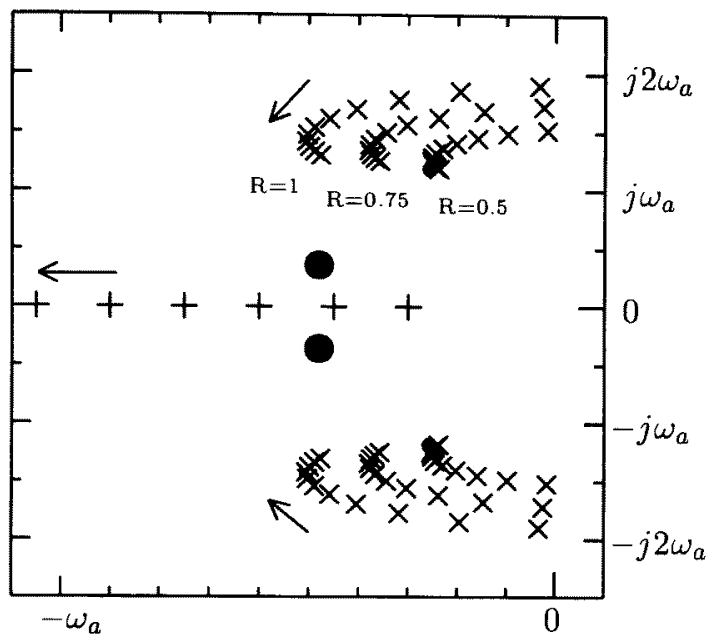

Fig. 2. Pole locations in the case of $1 / T_{d}=3 \omega_{a}$

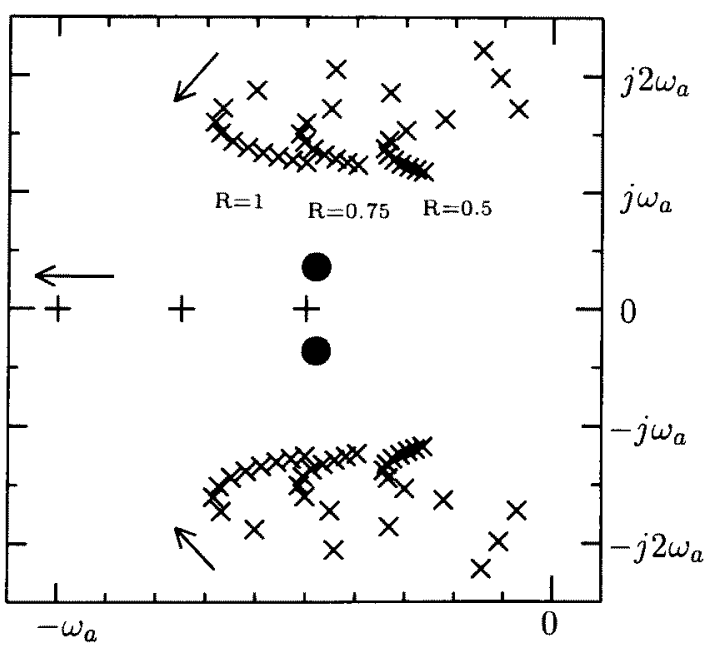

Fig. 3. Pole locations in the case of $1 / T_{d}=5 \omega_{a}$

In the case of $R=0.5$, the PID controller proposed by authors ${ }^{(2)}$ is tested for different time constant. The step responses are given in Fig.4, where $\omega_{a}$ is normalized as $1 \mathrm{rad} / \mathrm{s}$. It is shown that the filter brings the dynamic response worse, in particular, for $1 / T_{d}<5 \omega_{a}$.

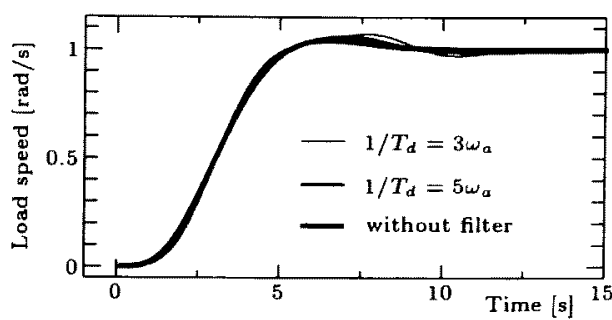

Fig. 4. PID control with or without filter

\section{Conclusions}

The speed servo system with PID controller plus a lowpass filter was analyzed. It was shown that the effect of the filter may be neglected without clearly losing the analysis accuracy when the bandwidth of the filter is 5 times wider over the antiresonant frequency. In fact, the condition is often approved by many industrial motor drive systems.

If the above condition is destroyed, the effect of the filter should be considered. Based on the extended parameter plane method, the system analysis was presented.

(Manuscript received March 25, 1999, revised April $13,1999)$

\section{References}

(1) G.Zhang and J.Furusho, "Systematic Analysis and Design on Two-Inertia System", Tran. SICE Japan, Vol.35, No.1, pp.105-112, 1999

(2) G.Zhang and J.Furusho, "A Practical PID Controller Design for Speed Servo System", Tran. IEE Japan, Vol.118-D, No.9, pp.1095-1096, 1998

(3) S.Manabe, "Controller Design of Two-Mass Resonant System by Coefficient Diagram Method", Tran, IEE Japan, Vol.118D, No.1, pp.58-66, 1998 (in japanese)

(4) Y.Hori, "Control of 2-Inertia System by a PID Controller", Tran. IEE Japan, Vol.115-D, No.1, pp.86-87, 1995 (in japanese)

(5) Y.Matsui, "Speed Control of 2-Mass System by PID Controller", Trans. IEE Japan, Vol.116-D, No.4, pp.441-447,1996 (in japanese)

(6) G.Zhang and J.Furusho, "PID Controller Design for Flexible Joint Robots", Proc. of JSME D \& D '99 6th Symposium on Motion and Vibration Control, pp.284-289, March 18-19, 1999, Chiba, Japan

Dr.Guoguang Zhang is a research associate of Osaka University. His research interests are motion control, vibration control and robotics.

Dr.Junji Furusho is a professor of the Department of Computer-Controlled Mechanical Systems at Osaka University. His research interests include robotics, motion control of mechanical systems and force display devices using intelligent fluids. 